\title{
Deriving permeability distributions from fractal Gaussian tracer returns.
}

\author{
GJ Weir ${ }^{1}$
}

Received 09 November 2017; Revised 17 May 2018

\begin{abstract}
Tracer returns in geothermal fields yield information about the connectivity between injection and production wells. We derive the equivalence between tracer returns described by a fractal Gaussian distribution, where diffusivity is scaled linearly with time, and tracer returns implied by one-sided Gaussian distributions of permeability. In this case, asymptotic tracer returns decay as the inverse square of time, and tracer returns are higher than predicted by methods assuming that asymptotic tracer returns decay exponentially with time.
\end{abstract}

\section{Contents}

\section{Introduction}

C206

DoI:10.21914/anziamj.v59i0.12611, (C) Austral. Mathematical Soc. 2018. Published September 25, 2018, as part of the Proceedings of the 13th Biennial Engineering Mathematics and Applications Conference. ISSN 1445-8810. (Print two pages per sheet of paper.) Copies of this article must not be made otherwise available on the internet; instead link directly to the DOI for this article. 
2 Fractal Gaussian

C208

3 Permeability structure

C210

4 Estimating tracer returns

C211

5 Summary

C213

References

C213

\section{Introduction}

Geothermal energy provides energy for both electricity production and as a direct heat source. It is often a sustainable energy source, and can play an important role in reducing greenhouse gas emissions. In New Zealand, for example, geothermal energy provides around $12 \%$ of electricity production, and has helped in replacing coal as an energy source. The International Geothermal Association (IGA) website provides an international and up-todate summary of geothermal production and use.

Geothermal fluids need to be reinjected into the earth, after energy extraction has occurred, to safeguard environmental water supplies. Reinjecting relatively cold fluid into a hot geothermal field can degrade the reservoir. Tracer tests provide information on connectivity between injection and production wells, and allow an assessment of the suitability of wells as injectors.

A typical injection experiment involves use of several injection and production wells. A different tracer is often used for each injection well, allowing the connectivity between each injection and each production well in the experiment to be assessed. It is not unusual for significant amounts of tracer from an injection well to be recovered at a production well, located around a kilometre away, within two months. 
Since tracer experiments are typically performed during standard operating conditions, the fluid recovered from a production well is reinjected, after moving through the geothermal power plant, and in this way, any recovered tracer again moves towards the production wells, and will be counted twice as it again arrives at the production well. After a significant amount of tracer has been counted twice at the production wells, it is not unusual for recorded tracer concentrations to tend towards a constant background value.

Standard industry analysis of tracer returns [3] proceed by identifying (usually by eye) when the first arrival part of the tracer returns begin to be influenced by multiple tracer returns. The total mass of tracer recovered up to this time is then calculated, and the early part of the tracer return is extrapolated into the future. The corresponding mass of extrapolated tracer is added to the earlier calculation, yielding the total mass of tracer recovered. The extrapolated function used in standard industry analyses is the unique decreasing exponential function of time, which joins continuously and first differentiably, to the early tracer profile, at the join point.

To the author's knowledge, such analyses have never yielded a $100 \%$ tracer recovery. Kristjansson et al.[4] report tracer recovery from the Icelandic injection well $\mathrm{HN}-10$ yielding $1 \%$ recovery, while $\mathrm{HN}-17$ in the same geothermal field yielded $58 \%$ recovery. The author has seen examples of around $85 \%$ tracer recovery from some unpublished records.

The standard industry analysis outlined above is essentially model independent, and infers tracer mass recovered directly from the experimental record. In contrast, many authors have used porous media theory, to infer and fit mathematical models to experimental tracer returns. Several hundred papers on analysing tracer returns are held in the IGA database, many of which are also contained in the Proceedings of the Stanford and New Zealand Geothermal Workshops, and many other high quality examples of tracer returns are held in confidential industry databases.

Many hundreds of tracer tests have also been conducted in groundwater reservoirs, as well as in rivers. Because of the relatively shallow depth of 
flow, it has been possible to sample these flows spatially. These observations have shown that many groundwater tracer profiles in space approximate a Gaussian distribution, in which the diffusivity varies linearly with the length scale of the experiment, and some with elapsed time [5, 6].

The aim of this paper is to assume this linearity (fractal) assumption for diffusivity found in groundwater tracer flows, and to apply it to geothermal tracer returns. We assume that injection and production rates are constant, which excludes back-flow tracer tests [7]; that tracer is not sorbed [8] onto rock; and that tracer follows approximately along a common flow path.

We identify two asymptotic regimes: one which decreases exponentially with time; and one which decreases as the inverse square of time. We find field data supports the later case. Because we have used analytical methods, the fractal Gaussian can be inverted exactly to show that the corresponding permeability distribution is a one-sided Gaussian. Finally, we show that the standard method of analysis can significantly underestimates tracer returns.

\section{Fractal Gaussian}

Tracer concentrations in a fluid flowing laminarly in a pipe obey the asymptotic Taylor law [9]

$$
C=\frac{M}{\sqrt{2 \pi \mathrm{Dt}}} \exp \left[-\frac{(x-u t)^{2}}{2 \mathrm{Dt}}\right],
$$

where $C$ is tracer concentration per pipe length $\left(\mathrm{kg} \mathrm{m}^{-1}\right), M$ is total tracer mass in the pipe $(\mathrm{kg}), \mathrm{D}$ is a constant diffusivity, $\mathrm{x}$ is position along the pipe from the injection point of the tracer, $u$ is the constant mean speed at which the tracer moves along the pipe, and $t$ is the time since the tracer was injected into the pipe.

Many groundwater tracer returns from the earth also approximate the concentration profile in (1), but in contrast, the diffusivity D scales linearly with 
the total length of travel of the tracer. Accepting that diffusivity will be estimated from data about the peak return, we could also conjecture that the diffusivity scales linearly with the time that the tracer has been in the earth, since the tracer is assumed to travel at the constant speed $u$. This suggests we consider the expression

$$
\mathrm{D}=\mathrm{u}_{1}^{2-\mathrm{a}} \chi^{\mathrm{a}} \mathrm{t}^{1-\mathrm{a}},
$$

where $\boldsymbol{u}_{1}$ has the dimensions of speed, and $0 \leqslant a<2$ is non-dimensional.

We will call a tracer profile a fractal Gaussian if it is obtained by substituting (2) into (1). Doing this yields

$$
\begin{gathered}
f d \Psi=-\frac{K d \Psi}{\sqrt{2 \pi \Psi^{a}}} \exp \left[-\frac{\left(\Psi^{1-\frac{a}{2}}-\frac{u}{u_{1}} \Psi^{-\frac{a}{2}}\right)^{2}}{2}\right], \\
\Psi=\frac{x}{u_{1} t} .
\end{gathered}
$$

The fractal Gaussian in (3) is now a function of the convective variable $\Psi$ in (4), rather than the diffusive variable $x^{2} t^{-1}$ in (1). The negative sign in (3) is introduced so that a positive sign is achieved when we let $t$ be the variable, $\mathrm{K}$ is a constant, and $\mathrm{fd} \Psi$ is the tracer return $(\mathrm{kg})$ obtained in an increment of $d \Psi$.

For small time $t, \Psi$ is very large, and as $1-\frac{a}{2}$ is always positive, $f$ is exponentially small for early times, showing that one needs to wait a finite interval of time before significant amounts of tracer arrive from the origin to the observation point at $x$. For very long times, $\Psi$ is very small, and the argument of the exponential in (3) varies as $-\left(\mathfrak{u} / \mathbf{u}_{1}\right)^{2-a} \mathbf{t}^{a} / 2$, showing that $\mathbf{f}$ is exponentially small for long times, unless $a=0$.

When $\mathbf{a}=0$, and time is used as the variable, (3) simplifies to

$$
f d t=\frac{K x d t}{u_{1} \sqrt{2 \pi} t^{2}} \exp \left[-\frac{\left(\frac{x}{u_{1} t}-\frac{u}{u_{1}}\right)^{2}}{2}\right],
$$


showing that $f$ decreases asymptotically as the inverse square in time. Several reports of long-tailed tracer returns have been reported from field trials [2, 5], and modelled using non-local effects [11].

It is not always possible to observe the asymptotic behaviour in geothermal tracer returns, because often multiple returns of tracer establish a background of tracer, which swamps observation of the tracer tail. Weir [12] has identified two geothermal tracer profiles with clear tails, and shown that (5), suitably parametrised, provides an excellent fit to the corresponding tracer profiles, and accordingly that the asymptotic tracer profiles decrease as $t^{-2}$. Then, at least for these two cases, the physically relevant tracer profiles, which are fractal Gaussian, are those with $a=0$, and $D=u_{1}^{2} t$.

In the special case that $\mathfrak{u}<<\mathfrak{u}_{1}$, (5) simplifies to

$$
f=\frac{2 M \tau}{\sqrt{\pi} t^{2}} \exp \left[-\left(\frac{\tau}{t}\right)^{2}\right],
$$

where $M$ is the total mass of tracer,

$$
\int_{0}^{\infty} f d t=M,
$$

and $\tau$ is the time that $f$ is maximum.

\section{Permeability structure}

The tracer profiles considered in the previous section were wholly empirical, being obtained by imposing a fractal structure on the Gaussian function. However, in all likelihood, there are no physical structures fractally distributed within the earth, which impose fractal tracer returns.

It is well known [10] from Horton's Laws that surface hydrological flows are fractal. As a result, large river valleys form far from river sources. There is no 
equivalent to these surface structures subsurface. Indeed, fractal behaviour in tracer profiles will develop even if the injection and production wells are interchanged. There is no equivalent to this in surface flows.

The permeability of rock [1] must have very special properties, therefore, if fractal behaviour is to emerge naturally in subsurface flows. The implied permeability structure is fixed, once the tracer profile is given. We illustrate this by using (6).

Consider the one-sided Gaussian probability distribution of permeability,

$$
p d k=\frac{2 d k}{\sqrt{\pi} k_{0}} \exp \left[-\left(\frac{k}{k_{0}}\right)^{2}\right], \quad<p>=1, \quad<k p>=\frac{k_{0}}{\sqrt{\pi}},
$$

where $k_{0}$ is a constant, $0 \leqslant k \leqslant \infty$ is the permeability variable, and $<\mathrm{p}\rangle=\int_{0}^{\infty} \mathrm{pdk}$. Then we note that

$$
f d t=-M p\left(k=\frac{k_{0} \tau}{t}\right) d\left(\frac{k_{0} \tau}{t}\right),
$$

exactly maps (8) into (6). Because of the inversion in (9), the probability function in (8) has all of its moments $\left\langle k^{n} p>\right.$ bounded, but all moments $<$ $t^{n} f>$ (except the zero moment, with $n=0$ ) of the corresponding probability function in (9) are unbounded.

\section{Estimating tracer returns}

Decades of analysis of tracer returns from geothermal fields has led to the widespread view that all of injected tracer is never recovered during tracer tests. For example, there are very few tracer tests which have yielded over $85 \%$ returns. The remaining $15 \%$ or more of tracer then must have remained within the earth. 
This is a strange result, since geothermal production wells induce flows which completely dominate background or natural flows. For example, production wells can induce flows which travel over a kilometre in a month, whereas natural flows may only travel tens of metres a year. This suggests that injected fluid should find its way from the injection to the production wells relatively easily, and with it the tracer. The implication is that at least some of the tracer must have attached to solid surfaces within the earth.

However, tracer attachment to rock should be a reversible process for at least some tracer molecules. Then tracer would move from the high concentrations in injected fluid to the rock, but after the peak tracer concentrations have past, the tracer should detach from the rock, and resume its passage to the production wells. This suggests that essentially all of the injected tracer could be recovered from the production wells, if the interaction of tracer with rock is a reversible process.

Contradicting this viewpoint is the observation that geothermal tracer experiments apparently do not yield total recovery. One likely implication is that some part of the tracer interaction with the rock is always effectively irreversible.

However, another interpretation of these total tracer returns is that the missing tracer is in the tails of the tracer distribution, and that current extrapolation methods significantly underestimate tracer returns. For example, consider extrapolating (6) from $t=2.5 \tau$. The extrapolated mass then equals $M \operatorname{erf}(.4) \simeq 0.43 \mathrm{M}$. However, fitting a decreasing exponential function of time yields the extrapolated mass $[M \exp (-1 / 6.25)] /(2.1 \sqrt{\pi}) \simeq 0.29 \mathrm{M}$. In this case, the standard extrapolation method has missed $0.14 \mathrm{M}$ of the tracer mass, and could explain current low estimates for tracer returns.

Which, if any, of these two viewpoints is correct, remains an open question. 


\section{Summary}

We proved that fractal Gaussians have two asymptotic behaviours: either decreasing exponentially with time, or decreasing as the inverse square of time. The latter class appear to apply to (at least some) geothermal tracer returns, and correspond uniquely to a one-sided Gaussian distribution of permeability.

The algebraic decay of these theoretical tracer returns predict much greater recovery of tracer than current standard methods, and may explain why current extrapolation methods never predict total tracer recovery. However, tracer returns, as with geological structures, are highly variable, and specific examples are likely to arise to contradict the key assumptions in any specific theory of tracer returns.

\section{References}

[1] J. Bear Dynamics of Fluids in Porous Media. American Elsevier, New York, 1972. C211

[2] M. W. Becker, A. M. Shapiro Tracer transport in fractured crystalline rock: Evidence of nondiffusive breakthrough tailing. Water Resour Res, 36(7), 1677-1686, 2000. doi:10.1029/2000WR900080. C210

[3] M. A. Grant, I. G. Donaldson, P. F Bixley Geothermal reservoir engineering. Academic Press, New York (1982) C207

[4] B. R. Kristjansson, G. Gudni Axelsson, G. Gunnarsson, I. Gunnarsson, F. Finnbogi Oskarsson, Comprehensive Tracer Testing in PROCEEDINGS, 41st Workshop on Geothermal Reservoir Engineering Stanford C207 
[5] R. Haggerty, S. A. McKenna, L. C. Meigs, On the late-time behavior of tracer test breakthrough curves. Water Resour Res 36(12), 3467-3479, 2000. doi:10.1029/2000WR900214 C208, C210

[6] A. Hunt, R. Ewing, B. Ghanbarian, Fractal Models of Porous Media. In: Percolation Theory for Flow in Porous Media. Lecture Notes in Physics, vol 880. Springer, 2014. C208

[7] I. Kocabas, R. N. Horne, Analysis of Injection-Backflow Tracer Tests in Fractured Geothermal Reservoirs. Proc. 12th Workshop on Geothermal Reservoir Engineering, Stanford University, Stanford, California, SGP-Tr-109, Jan 20-22, 1987. C208

[8] P. Rose, K. Leecaster, S. Clausen, R. Sanjuan,M. Ames, P. Reimus, M. Williams, V. Vermeul, D. Benoit, A tracer test at Proc. 37th Workshop on Geothermal Reservoir Engineering, Stanford University, Jan. 30 - Feb. 1, 2012. C208

[9] D. A. Nield, A. Bejin, Convection in Porous Media. 2nd edn. Springer, New York (1999) C208

[10] A. E. Scheidegger, Horton's laws of stream lengths and drainage areas. Water Resources Res, 4(5), 1015-1021, 1968. doi:10.1029/WR004i005p01015. C210

[11] R. Schumer, D. A. Benson, M. M. Meerschaert, B. Baeumer Fractal mobile/immobile solute transport. Water Resources Res, 39(10), 1296, 2003. doi:10.1029/2003WR002141 C210

[12] G. Weir, J. Burnell, Analysing tracer returns from geothermal reservoirs. In: Proceedings of 30th NZ Geothermal Workshop, Auckland, New Zealand, pp. 24 - 26, 2014. C210 


\section{Author address}

1. GJ Weir, Institute of Fundamental Sciences, Massey University, Palmerston North, New Zealand.

mailto:grahamweir@xtra.co.nz 